\title{
Fiber optic photoacoustic probe with ultrasonic tracking for guiding minimally invasive procedures
}

\author{
Wenfeng Xia ${ }^{1}$,Charles A. Mosse ${ }^{1}$, Richard J. Colchester ${ }^{1}$, Jean Martial Mari ${ }^{1}, 2$, Daniil I. \\ Nikitichev $^{1}$, Simeon J. West ${ }^{3}$, Sebastien Ourselin ${ }^{1}$, Paul C. Beard ${ }^{1}$ and Adrien E. Desjardins ${ }^{1}$ \\ ${ }^{1}$ Department of Medical Physics and Biomedical Engineering, University College London, \\ Gower street, London WC1E 6BT, United Kingdom; \\ ${ }^{2}$ GePaSud, University of French Polynesia, Faa'a, French Polynesia; \\ ${ }^{3}$ Main Theatres, Maple Bridge Link Corridor, Podium 3, University College Hospital, 235 \\ Euston Rd, London NW1 2BU, United Kingdom;
}

\begin{abstract}
In a wide range of clinical procedures, accurate placement of medical devices such as needles and catheters is critical to optimize patient outcomes. Ultrasound imaging is often used to guide minimally invasive procedures, as it can provide real-time visualization of patient anatomy and medical devices. However, this modality can provide low image contrast for soft tissues, and poor visualization of medical devices that are steeply angled with respect to the incoming ultrasound beams. Photoacoustic sensors can provide information about the spatial distributions of tissue chromophores that could be valuable for guiding minimally invasive procedures. In this study, a system for guiding minimally invasive procedures using photoacoustic sensing was developed. This system included a miniature photoacoustic probe with three optical fibers: one with a bare end for photoacoustic excitation of tissue, a second for photoacoustic excitation of an optically absorbing coating at the distal end to transmit ultrasound, and a third with a Fabry-Pérot cavity at the distal end for receiving ultrasound. The position of the photoacoustic probe was determined with ultrasonic tracking, which involved transmitting pulses from a linear-array ultrasound imaging probe at the tissue surface, and receiving them with the fiber-optic ultrasound receiver in the photoacoustic probe. The axial resolution of photoacoustic sensing was better than $70 \mu \mathrm{m}$, and the tracking accuracy was better than $1 \mathrm{~mm}$ in both axial and lateral dimensions. By translating the photoacoustic probe, depth scans were obtained from different spatial positions, and two-dimensional images were reconstructed using a frequency-domain algorithm.
\end{abstract}

KEYWORDS: fiber optic; photoacoustic imaging; ultrasound imaging; ultrasonic tracking; minimally invasive procedures

\section{INTRODUCTION}

In a wide range of clinical procedures, accurate placement of medical devices such as needles and catheters is critical to optimize patient outcomes. Ultrasound imaging is often used to guide minimally invasive procedures, as it can provide real-time visualization of patient anatomy and medical devices. However, this modality can provide low image contrast for soft tissues such as blood vessels, nerves, and tumors in some contexts. Furthermore, the visualization of medical devices such as needles and catheters can be challenging, with poor echogenicity when the devices are steeply angled with respect to the incoming ultrasound beams [1]. Inaccurate placement of devices can result in life-threatening complications.

Reflectance spectroscopy probes that are integrated into needles [2,3] and catheters [4] can provide information about the spatial distributions of tissue chromophore concentrations such as hemoglobin and lipid that is complementary to conventional B-mode ultrasound images. In a typical implementation, light is delivered to

Address all correspondence to: Wenfeng Xia, Department of Medical Physics and Biomedical Engineering, University College London, Malet Place Engineering Building, London WC1E 6BT, United Kingdom; Tel: +44 20 76790300; E-mail: wenfeng.xia@ucl.ac.uk.

Opto-Acoustic Methods and Applications in Biophotonics II, edited by Vasilis Ntziachristos, Roger Zemp, Proc. of SPIE Vol. 9539, 95390K · @ 2015 SPIE · CCC code: 1605-7422/15/\$18 · doi: 10.1117/12.2182647 
tissue with one optical fiber and a portion of the reflected light is received with one or more adjacent fibers. However, obtaining spatially-resolved information with reflectance spectroscopy probes is challenging, particularly with small numbers of optical fibers.

Photoacoustic sensors can provide information about tissue that could be valuable for guiding minimally invasive procedures. With photoacoustic sensing, pulsed or temporally modulated excitation light is delivered into tissue or to an optically absorbing coating. In the former case, ultrasound is generated when tissue chromophores absorb excitation light; in the latter, ultrasound is generated by the optically absorbing coating and ultrasonic reflections in tissue are received. Depending on how the ultrasound waves are received and how the excitation light is delivered, a photoacoustic sensor can either provide one-dimensional depth scans or two-/three-dimensional images. When integrated into medical devices, photoacoustic sensors could be used to detect and spatially-localize specific tissue structures. In this study, an all fiber-optic system for guiding minimally invasive procedures using photoacoustic and ultrasound sensing was developed. The potential of the system's ability to acquire co-registered photoacoustic and ultrasound images was demonstrated using a tissue-mimicking phantom. Further, the tip of the fiber-optic probe was successfully tracked using a clinical ultrasound array in a water phantom. The results suggest that the system holds strong potential to guide various minimally invasive procedures.

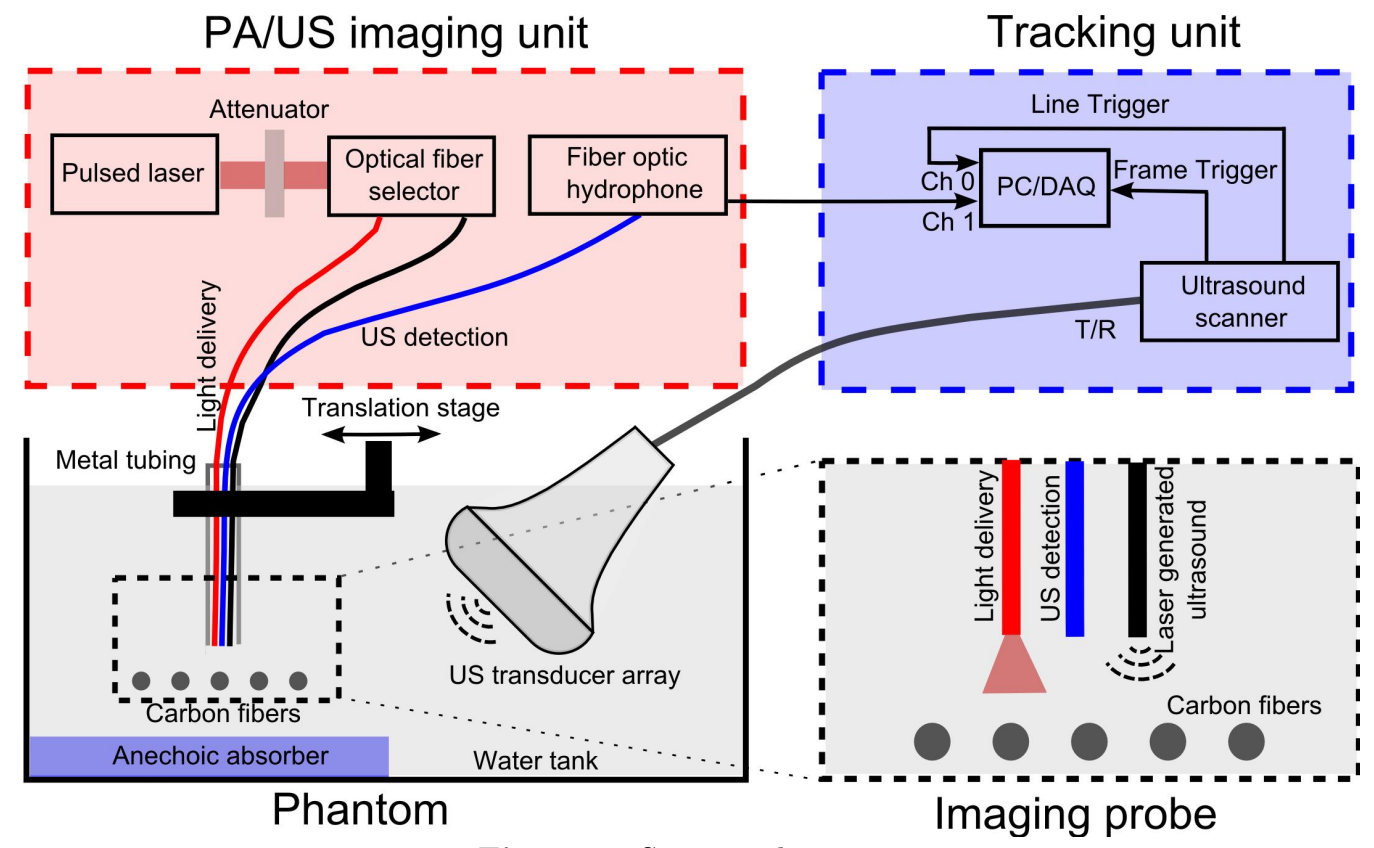

Figure 1: System schematic.

\section{MATERIALS AND METHODS}

The system included a miniature photoacoustic probe with three optical fibers: one with a bare distal end for delivering pulsed excitation light to tissue, a second with an optically absorbing coating at the distal end for transmitting ultrasound, and a third with a Fabry-Pérot cavity at the distal end for receiving ultrasound (Figure 1). The optically absorbing coating was a composite of functionalized carbon nanotubes and polydimethylsiloxane (PDMS), as previously described [5]. The Fabry-Pérot cavity comprised a transparent polymer positioned between two reflective surfaces [6]. The position of the photoacoustic probe was determined with ultrasonic tracking, which involved delivering pulses an ultrasound imaging probe, and receiving them with the fiber-optic ultrasound receiver in the photoacoustic probe [7].

A photoacoustic/ultrasound imaging unit delivered excitation light for generating photoacoustic signals and measured reflectivity from the Fabry-Pérot cavity for receiving ultrasound. Excitation light pulses at $1064 \mathrm{~nm}$ were delivered by a fiber coupled Nd:YAG laser (SPOT-10-500-1064, Elforlight, UK). Continuous-wave light was provided to the Fabry-Pérot cavity by a wavelength-tunable laser (Tunics T100S-HP CL, Yenista Optics, 
France), with the wavelength chosen for maximal sensitivity to impinging ultrasound waves. The switch between photoacoustic and ultrasound imaging is achieved by a manual optical fiber selector.

The primary component of the tracking unit was a clinical US imaging system (SonixMDP, Analogic Ultrasound, Richmond, BC, Canada) with a linear-array ultrasound imaging probe (L14-5, bandwidth: 5-14 MHz, Analogic Ultrasound, Richmond, BC, Canada). This imaging system, which was operated in research mode, was used to obtain conventional B-mode ultrasound images and to generate sequences of ultrasound pulses from individual transducer elements that were received by the fiber-optic ultrasound receiver in the photoacoustic probe. Assuming a constant ultrasound speed, the time-of-flights of the ultrasound transmissions are proportional to the distance between the receiver and the center of the transmitting elements. As such, an image of the receiver can be obtained by processing signals corresponding to transmissions from different transducer elements. An important advantage of this tracking method is that the conventional B-mode ultrasound image and the image of the receiver are inherently co-registered.
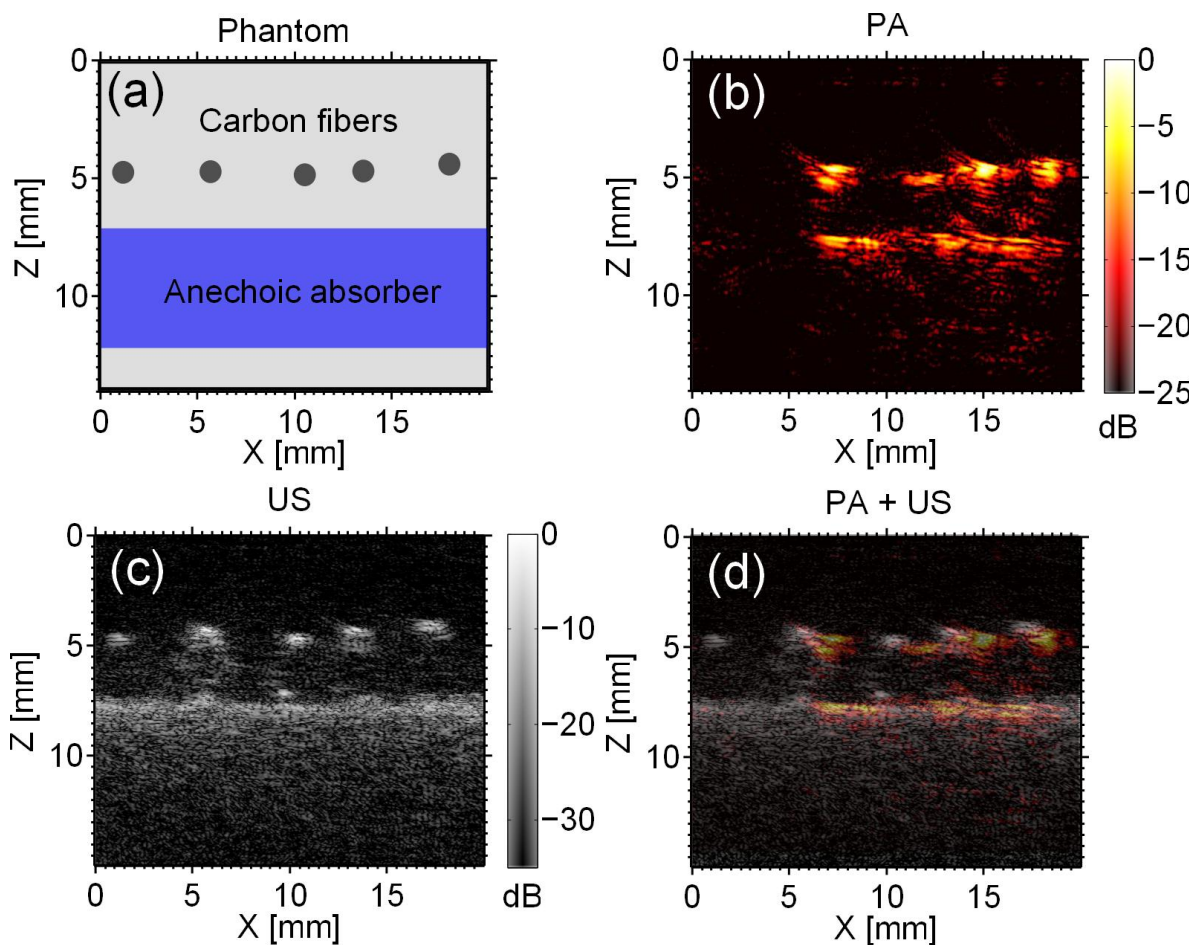

Figure 2: Co-registered images of a phantom consisting 5 carbon fiber targets positioned above an anechoic absorber (a), which were obtained with the photoacoustic probe. The photoacoustic (b) and ultrasound (c) images were reconstructed from a series of depth scans obtained with the photoacoustic probe at different spatial positions, and overlaid (d).

\section{RESULTS}

Photoacoustic imaging with the system was tested using a simple phantom (Figure 1). This phantom comprised 5 carbon fibers with $100 \mu \mathrm{m}$ diameters as imaging targets, which were aligned horizontally and positioned several millimetres above an anechoic acoustic absorber in a water bath (Figure 2a). The photoacoustic probe was mounted on mechanical translation stage so that its lateral position relative to the imaging targets could be varied. Photoacoustic and ultrasound images were obtained consecutively. The former were obtained with excitation light provided to tissue; the latter, with excitation light provided to the optically-absorbing coating. Both the photoacoustic and the ultrasound images were reconstructed offline using a frequency-domain algorithm [8] implemented with the k-Wave toolbox for Matlab [9].

The targets and the surface of the blue anechoic absorber were clearly visualized in the photoacoustic image (Figure 2(b)). The carbon fiber at $\mathrm{x}=1.5 \mathrm{~mm}$ did not appear in the photoacoustic image, which can be attributed 
to the lack of light illumination given that the probe was angled with respect to the phantom. However, all 5 carbon fibers were visualized in the ultrasound image (Figure 2(c)). The structure of the anechoic absorber was also apparent in the ultrasound image. Both photoacoustic and ultrasound images were spatially well co-registered (Figure 2(d)).

Ultrasonic tracking performance was assessed with the photoacoustic probe and the surface-mounted ultrasound imaging probe positioned in a water bath. When concatenated, a parabolic shape was observed from the fiber-optic receiver signals that corresponded to ultrasound transmissions from individual elements of the linear-array ultrasound imaging probe at the tissue surface (Figure 3a). A reconstructed image of the receiver typically comprised a single spot that was localized to within a spatial region smaller than $1 \times 1 \mathrm{~mm}^{2}$ (Figure $3 \mathrm{~b}$ ), which was confirmed by the axial and lateral profiles (Figures 3c-d).
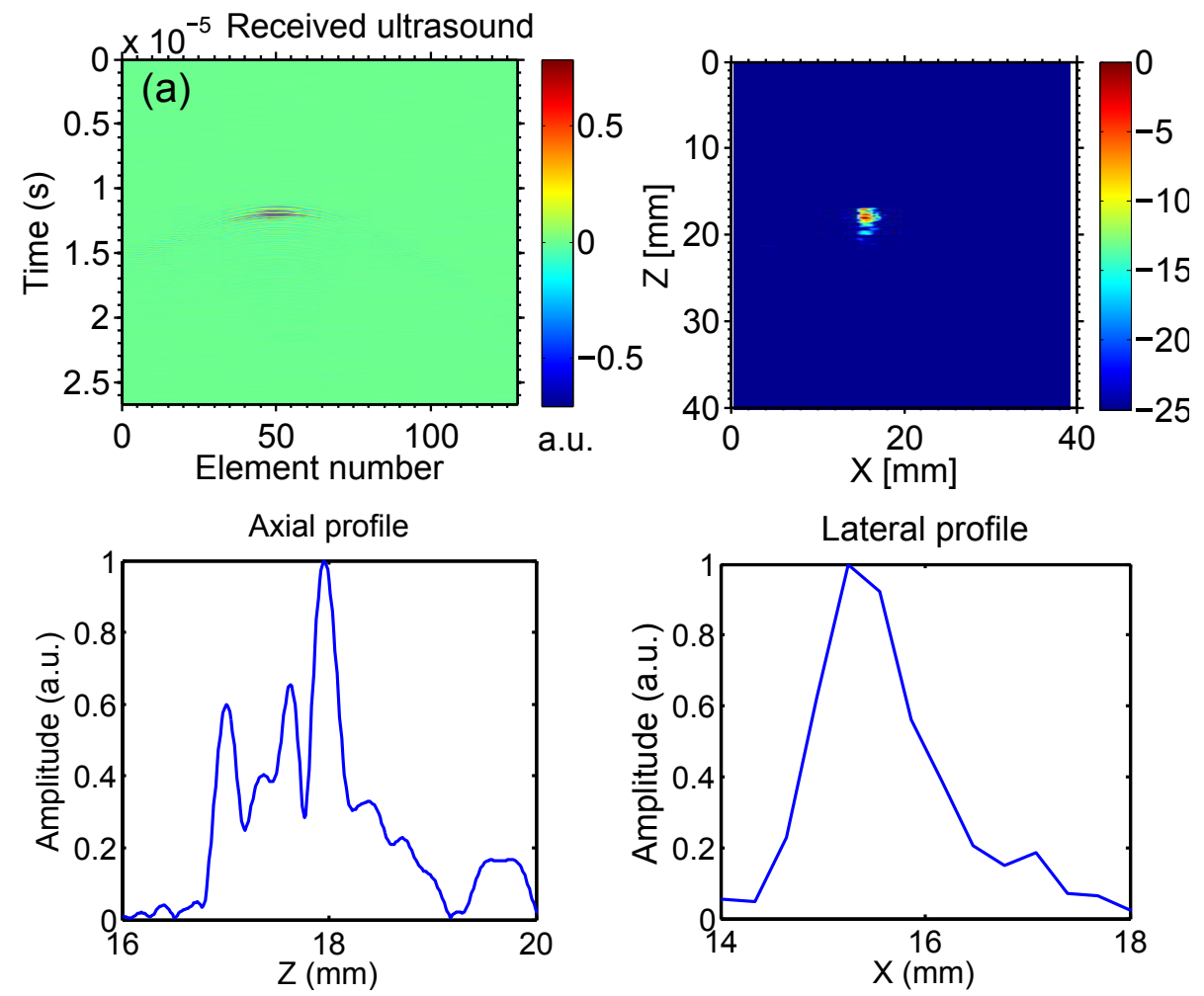

Figure 3: Ultrasonic tracking of the fiber optic hydrophone tip involved the reception of ultrasound pulses from a linear-array ultrasound imaging probe positioned at the surface of the tissue phantom. The raw signals (a) were reconstructed to obtain an image of the tip of the fiber-optic ultrasound receiver (b). The axial and lateral profiles of the reconstructed hydrophone tip are shown in (c) and (d) respectively.

\section{DISCUSSION AND CONCLUSION}

The system for guiding minimally invasive procedures that was developed in this study provides information that is complementary to conventional ultrasound and x-ray fluoroscopic images. Photoacoustic sensing performed with excitation of tissue provides information about the spatial distribution of specific chromophores. Ultrasound sensing, in which transmission across a broad frequency range is performed with photoacoustic excitation of an optically absorbing coating, can provide structural information with spatial resolution higher than those of ultrasound imaging probes positioned at the tissue surface. Ultrasound tracking of the photoacoustic probe using the fiber optic ultrasound receiver could allow for co-registration of signals obtained from spatial regions close to the photoacoustic probe with larger-scale, lower-resolution images obtained from linear-array ultrasound imaging probes at the tissue surface. Ultimately, the position information provided by ultrasound tracking could be used to reconstruct images from the photoacoustic probe signals in real-time during free-hand positioning in the human body. 


\section{ACKNOWLEDGMENTS}

This work was supported by an Innovative Engineering for Health award by the Wellcome Trust [WT101957] and the Engineering and Physical Sciences Research Council (EPSRC) [NS/A000027/1], by a Starting Grant from the European Research Council [ERC-2012-StG, Proposal 310970 MOPHIM], and by an EPSRC First Grant [EP/J010952/1].

\section{REFERENCES}

1. K. Chin, A. Perlas, V. Chan, and R. Brull, "Needle visualization in ultrasound-guided regional anesthesia: challenges and solutions," Reg. Anesth. Pain 33(6), 532-544, (2008).

2. A. E. Desjardins, M. van der Voort, R. Nachabe, S. Roggeveen, G. Lucassen, W. Bierhoff, B. H. W. Hendriks, M. Brynolf, and B. Holmstrom, "Needle stylet with integrated optical fibers for spectroscopic contrast during peripheral nerve blocks," J. Biomed. Opt. 16(7), 077004, (2011).

3. A. E. Desjardins, B. H. Hendriks, M. van der Voort, R. Nachab, W. Bierhoff, G. Braun, D. Babic, J. P. Rathmell, S. Holmin, M. Soderman, and B. Holmstrom, "Epidural needle with embedded optical fibers for spectroscopic differentiation of tissue: ex vivo feasibility study," Biomed. Opt. Express 2(6), 350-357, (2011).

4. S. H. Tabrizi, S. M. R. Aghamiri, F. Farzaneh, A. Amelink, H. J. C. M. Sterenborg, "Single fiber reflectance spectroscopy on cervical premalignancies: the potential for reduction of the number of unnecessary biopsies," J. Biomed. Opt. 18(1), 017002, (2013).

5. R. J. Colchester, C. A. Mosse, D. S. Bhachu, J. C. Bear,Claire J. Carmalt, I. P. Parkin, B. E. Treeby, I. Papakonstantinou, and A. E. Desjardins, "Laser-generated ultrasound with optical fibres using functionalised carbon nanotube composite coatings," Appl. Phys. Lett. 104, 173502, (2014).

6. E. Zhang, and P. C. Beard, "Characteristics of optimized fibre-optic ultrasound receivers for minimally invasive photoacoustic detection," Proc. of SPIE 9323, 932311, (2015).

7. J. M. Mari, S. West, P. C. Beard, A. E. Desjardins, "Needle-tip localization using an optical fibre hydrophone," Proc. of SPIE 8938, 893804, (2014).

8. Y. Xu, D. Feng, and L. V. Wang, "Exact frequency-domain reconstruction for thermoacoustic tomography I: Planar geometry," IEEE Trans. Med. Img. 21(7), 823-828, (2002).

9. B. E. Treeby, B. T. Cox, "k-Wave: MATLAB toolbox for the simulation and reconstruction of photoacoustic wave fields," J. Biomed. Opt. 15(2), 021314, (2010). 